\title{
The role of support and other factors in early breastfeeding cessation: an analysis of data from a maternity survey in England
}

\author{
Laura L Oakley ${ }^{1,2^{*}}$, Jane Henderson ${ }^{1}$, Maggie Redshaw ${ }^{1}$ and Maria A Quigley ${ }^{1}$
}

\begin{abstract}
Background: Although the majority of women in England initiate breastfeeding, approximately one third cease breastfeeding by six weeks and many of these women report they would like to have breastfed for longer.

Methods: Data from a survey of women $\geq 16$ years who gave birth to singleton term infants in 2009 in England; questionnaires were completed approximately three months postnatally. Logistic regression was used to investigate the association between postnatal support and other factors, and breastfeeding cessation at 10 days and six weeks. Population attributable fractions (PAFs) were calculated to estimate the relative contribution of breastfeeding support factors to overall breastfeeding cessation at these two time points.

Results: Of the 3840 women who initiated breastfeeding and reported timing of breastfeeding cessation, 13\% had stopped by 10 days; and of the 3354 women who were breastfeeding at 10 days, $17 \%$ had stopped by six weeks. Socio-demographic factors (maternal age, ethnicity, country of birth, deprivation, education) and antenatal feeding intention were all independently associated with breastfeeding cessation at 10 days and six weeks. Women who did not receive feeding advice or support from a parent or peer support group, voluntary organisation, or breastfeeding clinic were more likely to stop breastfeeding by 10 days. Perceived active support and encouragement from midwives was associated with a lower odds of breastfeeding cessation at both 10 days and six weeks. Estimated PAFs suggest that 34-59\% of breastfeeding cessations by 10 days could be avoided if more women in the study population received breastfeeding support.

Conclusion: Although multiple factors influence a mother's likelihood of continuing breastfeeding, it is clear that socio-demographic factors are strongly associated with breastfeeding continuation. However, there is evidence that breastfeeding support, including that delivered by peer or lay support workers, may have an important role in preventing cessations in the first few weeks.
\end{abstract}

Keywords: Breastfeeding, Epidemiology, Public health, Socio-economic

\section{Background}

The number of mothers in England who initiate breastfeeding has seen a small but steady increase over the last two decades [1] with data from the 2010 UK Infant Feeding Survey (IFS) indicating that $83 \%$ of mothers in England now initiate breastfeeding. However, discontinuation rates remain high in the early weeks: nearly

\footnotetext{
* Correspondence: laura.oakley@lshtm.ac.uk

${ }^{1}$ Policy Research Unit in Maternal Health and Care, National Perinatal Epidemiology Unit, University of Oxford, Old Road Campus, Headington, Oxford OX3 7LF, UK

${ }^{2}$ Department of Non-communicable Disease Epidemiology, London School of Hygiene and Tropical Medicine, Keppel St, London WC1E 7HT, UK
}

one in three mothers who initiate breastfeeding cease by six weeks [1].

The factors which influence whether or not a mother continues breastfeeding may differ from those which affect breastfeeding initiation. A number of studies conducted in the UK and other high-income settings have investigated the association between a range of factors and breastfeeding duration. These studies have identified a strong association between duration and antenatal feeding intention and attitudes, [2-5] and socio-demographic factors including ethnicity, [1,4,6,7] age,[1,2,4,8] maternal education $[4,8-10]$ and socio-economic or area deprivation status 
$[1,7,9,11,12]$. In addition, factors relating to maternity care and hospital infant feeding practices are associated with breastfeeding continuation, $[2,4,9]$ though an association with labour and birth factors is less clear $[2,6,8,13]$.

Evidence regarding the effect of breastfeeding support on breastfeeding duration is largely drawn from evaluations of relevant interventions. Although a recently updated Cochrane review suggests that breastfeeding support interventions appear to have a beneficial effect on increasing the number of mothers who breastfeed beyond the immediate postnatal period, [14] results from trials conducted in the UK so far do not provide support for this conclusion. Of the nine UK breastfeeding trials included in a recent narrative review, none reported significant improvement in breastfeeding outcomes, [15] and the five UK-based RCTs included in a systematic review of peer breastfeeding support all failed to demonstrate a significant effect on breastfeeding continuation [16]. Few observational studies have focused on the effect of breastfeeding support, though in a multivariable analysis of 2010 UK IFS data, awareness and/or use of breastfeeding support services was independently associated with breastfeeding continuation at two weeks but not at six weeks postpartum [1].

We used data from a recent national maternity survey to investigate factors associated with breastfeeding cessation in the first six weeks.

\section{Methods}

The objectives of our study were to investigate the factors associated with breastfeeding cessation at 10 days and six weeks, and to assess the relative contribution of breastfeeding support factors to overall breastfeeding cessation at these two time points.

\section{Study design}

We used data from a national survey of women conducted in England in 2010, designed to report on women's experiences of maternity care, health and wellbeing up to three months postnatally. A random sample of 10,000 women $\geq 16$ years who gave birth in a fortnight period in 2009 was identified by the Office for National Statistics (ONS) using birth registration data. Women whose babies had died were excluded from the sampling frame. Eligible women were sent a questionnaire, letter and information about the study in a range of languages when their babies were approximately three months old. After a series of tailored reminders (a reminder letter after two weeks, a further questionnaire after four weeks), 5,333 women (55.1\%) returned a usable questionnaire. The original survey was approved by the Trent Multi-Centre Research Ethics Committee. Further details of the study methodology are published elsewhere [17].
Our analysis was restricted to women who gave birth to singleton infants at term gestation ( $\geq 37$ weeks gestation). Multiple births and preterm infants were excluded due to small numbers and more complex feeding patterns which may not have been captured in our questionnaire.

\section{Measures}

Breastfeeding cessation was assessed among women who reported initiating breastfeeding, where initiation was defined as the baby receiving any breast milk (hereafter referred to being breastfed), either exclusively or in conjunction with the use of infant formula, in the first few days after birth. Cessation was defined as the point at which the baby no longer received any breast milk. We focused on cessation at two time points: 10 days (among those initiating breastfeeding) and six weeks (among those still breastfeeding at 10 days). These time points were chosen to reflect key points in the postnatal period, with discharge from midwife care usually occurring by day 10 and the routine infant check scheduled to take place at 6-8 weeks.

We included a range of explanatory variables which were grouped as socio-demographic, antenatal and birth, or postnatal support.

The socio-demographic variables included area-based deprivation (Index of Multiple Deprivation quintile IMD [18]), age of woman, age completed full-time education, cohabitation status, parity, ethnicity and country of birth. Antenatal and birth factors comprised antenatal feeding intention, whether the midwife had discussed infant feeding during antenatal care, gestational age at birth, birthweight, mode of birth, skin to skin after birth, duration of labour, length of postnatal hospital stay, neonatal unit (NNU) admission, and maternal physical health in the first few days. The postnatal support variables included: frequency of postnatal midwife visits, continuity of carer postnatally, baby's age at last midwife visit, and a number of questions addressing perceived quality of midwife or carer help or advice regarding feeding and the source of any feeding support received (wording for these questions given in Table 1).

With the exception of IMD which was provided by the ONS, all variables were self-report measures taken from survey data.

\section{Statistical analysis}

Logistic regression modelling was conducted in three stages due to the large number of explanatory variables. Firstly, unadjusted odds ratios (OR) were calculated for the association between each explanatory variable and breastfeeding cessation at both 10 days and 6 weeks. Secondly, these ORs were adjusted for other factors in the same group (socio-demographic, antenatal and birth, postnatal support). Finally, the ORs were adjusted for all 


\section{Table 1 Breastfeeding support questions}

\begin{tabular}{|c|c|}
\hline \multirow{2}{*}{$\begin{array}{l}\text { Thinking about feeding your baby, did } \\
\text { you feel that midwives and other } \\
\text { carers gave you: }\end{array}$} & Yes, always \\
\hline & Yes, generally \\
\hline a) Consistent advice? & No \\
\hline b) Practical help? & Don't know \\
\hline c) Active support and encouragement? & Didn't want this \\
\hline
\end{tabular}

(Please tick one answer for each of $a$ ), b) and c))

\begin{tabular}{ll}
\hline Who helped or advised you with & Health professions \\
feeding your baby? & Partner/friend/relative \\
& Parent support or peer group \\
& Voluntary organisation \\
& Breastfeeding clinic \\
& I was not given any help or \\
& advice \\
& I did not need help or advice \\
& (Please tick all that apply) \\
\hline Since your baby was born have the & Was available \\
following been available or used by you? & \\
a) A 'baby cafér" & Have attended or used \\
& (Please tick one answer or leave \\
& blank if service not available)
\end{tabular}

'baby cafés' are a national network of drop-ins providing breastfeeding information and support, led by a health professional or breastfeeding counsellor.

explanatory variables together. At each stage explanatory variables were only retained if they were independently associated with breastfeeding cessation, defined as at least one odds ratio at $\mathrm{p}<0.05$ (Wald test). Therefore, variables that did not significantly change the fit of the final model were dropped.

We calculated population attributable fractions (PAFs) for breastfeeding support variables independently associated with breastfeeding cessation at either of the two time points. PAFS were calculated using the formula PAF = $\mathrm{p}(\mathrm{OR}-1) / \mathrm{OR}$, where 'p' was the proportion of those who had ceased breastfeeding who reported receiving the specified support and OR was the adjusted odds ratio. Confidence intervals for PAFs were calculated using the formula suggested by Greenland [19].

Univariable analysis was conducted using all available data. For multivariable modelling, analysis was restricted to those observations with complete data on all variables included in the multivariable model. For four variables with $3-15 \%$ missing values (country of birth, baby's age at last MW contact, NNU admission, length of postnatal hospital stay) a further category of 'missing' was included to enable the variable to be included in modelling without substantial reduction in sample size.

IMD quintile was associated with non-response, [17] hence we weighted the sample for non-response using
IMD quintile. All reported frequencies are unweighted and all reported percentages weighted unless otherwise specified. Descriptive analysis is only presented for variables that were statistically significant $(\mathrm{p}<0.05)$ in multivariable analysis; additional results are presented as supplementary data (Additional file 1: Table S1).

All statistical analysis was conducted using the survey commands in Stata version 11.

\section{Results}

Data on initial feeding status were available for 4,818 women (Figure 1). Figure 2 shows the proportion of respondents in the survey who initiated breastfeeding and those still breastfeeding at intervals up to six weeks. The breastfeeding rates are almost identical to those observed in the IFS 2010.

\section{Breastfeeding cessation at 10 days}

Of the 3840 women who initiated breastfeeding and provided information on the timing of any breastfeeding cessation, 13\% $(\mathrm{n}=486)$ had stopped breastfeeding by 10 days (Table 2 ).

In univariable analysis, cessation at 10 days was significantly associated with all socio-demographic factors, and a number of antenatal and birth factors (antenatal feeding intention, antenatal discussion of infant feeding, labour duration, length of postnatal stay, and NNU admission) and support factors (baby's age at last contact with midwife, receiving feeding help or advice from a number of specific sources, needing any feeding support) (Tables 2 and 3). In addition, those women who reported that midwives did not give consistent advice regarding feeding, or did not provide active support and encouragement, were more likely to have stopped breastfeeding at 10 days.

In multivariable analysis, the patterns were similar. Most socio-demographic factors were independently associated with cessation at 10 days, although effects were somewhat attenuated by adjustment (Table 4). Area deprivation, younger age, White ethnicity, younger age at completion of full-time education, and UK birth all continued to be associated with increased odds of breastfeeding cessation at 10 days. Older age remained associated with a lower odds of cessation. Antenatal feeding intention remained strongly associated with breastfeeding cessation at 10 days, with those who intended to mixed feed, bottle feed, or with no firm intention all more likely to have ceased breastfeeding by 10 days. Duration of labour (specifically "no labour") and infant admission to NNU remained associated with a lower odds of cessation, and longer hospital postnatal stay continued to be associated with a higher odds of cessation. In terms of key support variables, women who did not receive feeding advice or support from a parent support/peer group, voluntary 


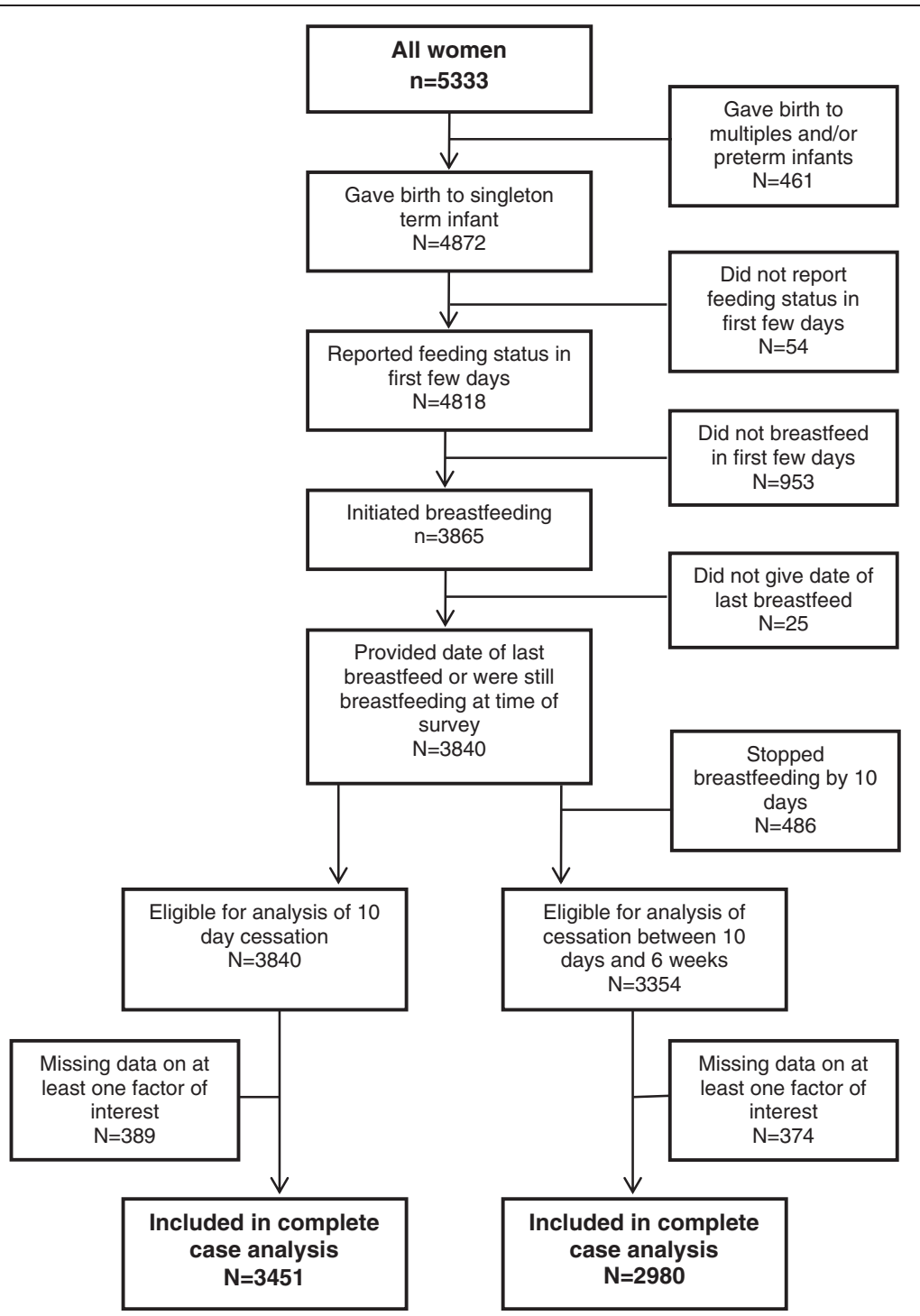

Figure 1 Flowchart of study sample.

organisation or breastfeeding clinic were more likely to stop breastfeeding by 10 days, with little change after adjustment for other factors (Table 5). Compared to women who reported that midwives 'always' gave active support and encouragement regarding feeding, those who said this support was 'generally' given or not given at all were more likely to have stopped breastfeeding at 10 days.

\section{Breastfeeding cessation at six weeks}

Of the 3354 women who were breastfeeding at 10 days, $17 \%(\mathrm{n}=551$ ) had stopped by six weeks (Table 2 ).

Breastfeeding cessation between 10 days and six weeks was associated with all socio-demographic factors in univariable analysis, with patterns similar to those observed in the analysis of breastfeeding cessation at 10 days (Table 2). Antenatal feeding intention was also strongly associated with cessation at six weeks in univariable analysis, as was gestation, type of birth, duration of labour, and maternal health in the first few days. In terms of support variables, only needing help and advice regarding feeding, attendance at a baby café, and quality of midwifery care regarding feeding (consistency of advice, practical help, active support and encouragement) were associated with a lower odds of breastfeeding cessation between 10 days and six weeks in univariable analysis (Table 3).

Compared to the analysis of breastfeeding cessation by 10 days, a smaller number of factors were independently associated with cessation between 10 days and six weeks (Tables 4 and 5). After adjustment, cessation by six weeks was lowest in older mothers, and highest in women living in the most deprived areas, who had a 


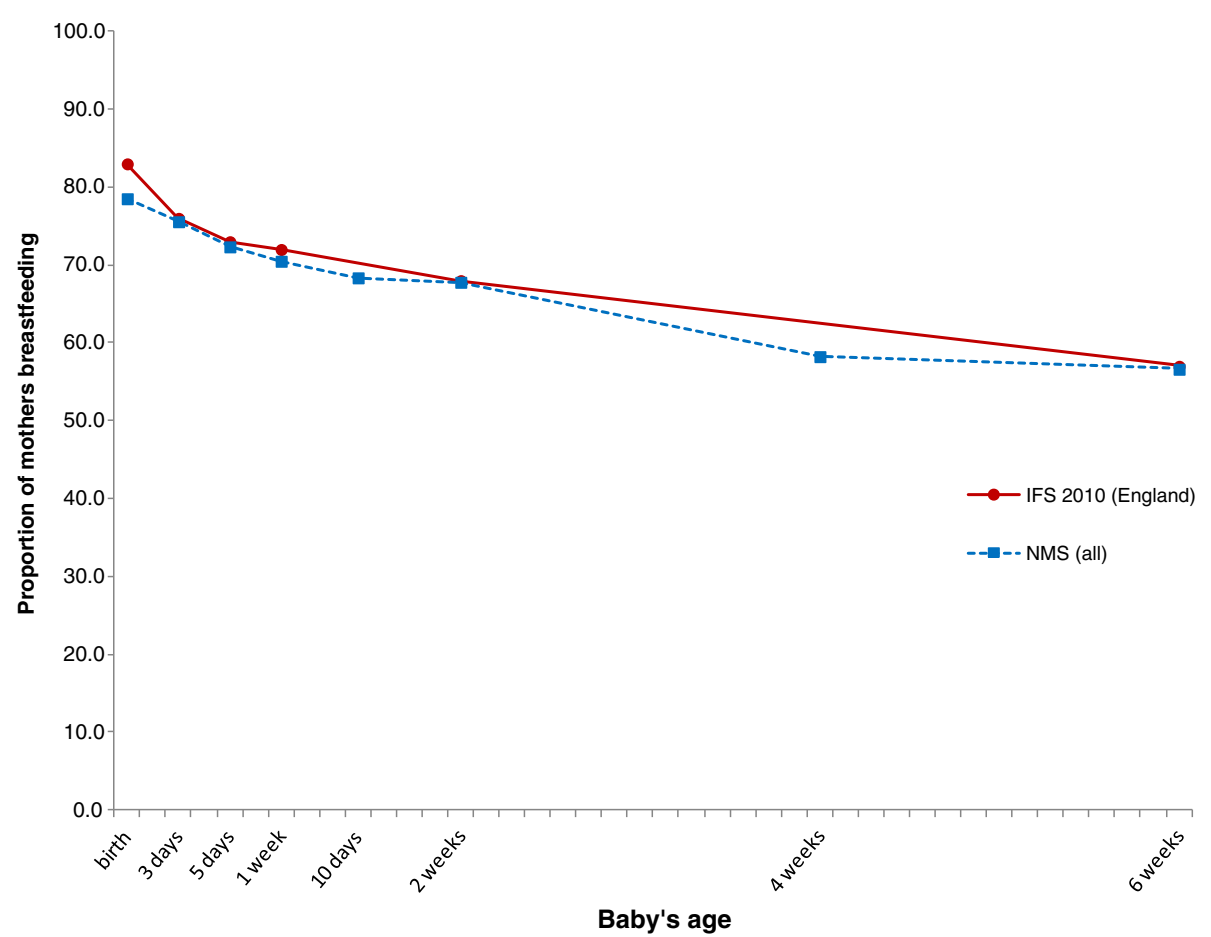

Figure 2 Breastfeeding initiation and duration: survey data and IFS 2010. Includes mothers who gave birth to preterm infants and/or multiples. ${ }^{\ddagger}$ Data for England only.

younger age at completing full-time education, who were White ethnicity, and those born in the UK (Table 4). Antenatal intention to formula feed continued to be significantly associated with increased cessation between 10 days and six weeks, as did higher gestation and planned caesarean. Attendance at a baby café remained in the multivariable model, with a higher odds of cessation for those reporting that this particular service was not available. Women who reported needing help and advice regarding feeding, and those who reported that midwives did not always give active support and encouragement regarding feeding, were more likely to have stopped breastfeeding by six weeks even after adjusting for other factors.

\section{Population attributable fractions}

We calculated PAFs for three variables independently associated with breastfeeding cessation at 10 days: whether women had received feeding help from a parent support or peer group; whether women had received feeding help from a voluntary organisation; and whether women had received feeding help from a breastfeeding clinic (Table 5). The PAFs for these three variables were 34.0\%, $58.9 \%$ and $58.1 \%$ respectively, suggesting that, assuming causality, $34-59 \%$ of breastfeeding cessations by 10 days could be avoided if more women in the study population received breastfeeding support.

\section{Discussion}

In our analysis of data from a recent national maternity survey, socio-demographic factors, feeding intention and breastfeeding support were all independently associated with breastfeeding cessation. Apart from breastfeeding support which was only independently associated with cessation by 10 days, there was little difference in the factors associated with cessation at 10 days and six weeks. Although a large number of explanatory variables were included in the univariable analysis and many of these showed associations with breastfeeding cessation, adjustment for other factors attenuated many of these associations.

A strong effect of socio-demographic factors on breastfeeding has been observed in numerous individual level studies $[1,2,4,6-11]$ and also by a recent area level analysis [20]. We found that younger women, women living in more deprived areas, those with lower levels of education, and White women were all more likely to discontinue breastfeeding. It is interesting that not only are these groups less likely to initiate breastfeeding, but that even if they initiate breastfeeding, they are less likely to continue to 10 days, and even if they are breastfeeding at 10 days, they are less likely to continue to six weeks, compared with their counterparts in other sociodemographic groups. Many previous studies investigating breastfeeding duration have included in their sample 
Table 2 Breastfeeding initiation and cessation by socio-demographic, antenatal and birth characteristics

\begin{tabular}{|c|c|c|c|c|c|}
\hline & \multirow{2}{*}{$\begin{array}{l}\text { All women } \\
n^{a}\end{array}$} & \multicolumn{2}{|c|}{$\begin{array}{c}\text { Initiated } \\
\text { breastfeeding }\end{array}$} & \multirow{2}{*}{$\begin{array}{l}\text { Stopped breastfeeding } \\
\text { by } 10 \text { days } \\
(\%)^{b, c}\end{array}$} & \multirow{2}{*}{$\begin{array}{c}\text { Stopped breastfeeding between } \\
10 \text { days and } 6 \text { weeks } \\
(\%)^{\text {b,d }}\end{array}$} \\
\hline & & $n^{a}$ & $(\%)^{\mathbf{b}}$ & & \\
\hline All women & 4818 & 3865 & $(79.1)$ & $(13.0)$ & $(16.7)$ \\
\hline \multicolumn{6}{|l|}{ Index of Multiple Deprivation } \\
\hline Quintile 1 (least deprived) & 965 & 829 & $(85.9)$ & $(10.0)$ & $(13.6)$ \\
\hline Quintile 2 & 954 & 801 & $(84.0)$ & $(11.6)$ & $(14.8)$ \\
\hline Quintile 3 & 1046 & 859 & $(82.1)$ & $(13.4)$ & (18.4) \\
\hline Quintile 4 & 896 & 700 & (78.1) & $(13.4)$ & $(17.9)$ \\
\hline Quintile 5 (most deprived) & 956 & 675 & (70.6) & $(15.5)$ & $(18.1)$ \\
\hline \multicolumn{6}{|l|}{ Age } \\
\hline $16-19$ & 137 & 61 & $(44.8)$ & (35.6) & (39.9) \\
\hline $20-24$ & 629 & 384 & $(60.1)$ & $(24.7)$ & $(26.7)$ \\
\hline $25-29$ & 1179 & 943 & $(79.2)$ & $(13.8)$ & $(18.3)$ \\
\hline $30-34$ & 1605 & 1390 & (85.9) & $(11.2)$ & $(16.3)$ \\
\hline $35-39$ & 1006 & 852 & $(84.3)$ & $(8.2)$ & $(11.3)$ \\
\hline$\geq 40$ & 238 & 215 & (89.6) & $(8.2)$ & (11.8) \\
\hline
\end{tabular}

Age completed full-time education

\begin{tabular}{|c|c|c|c|c|}
\hline$<17$ years & 1039 & 632 & $(59.0)$ & $(21.4)$ \\
\hline $17-18$ years & 1262 & 946 & $(74.2)$ & $(19.6)$ \\
\hline $19+$ years & 2408 & 2196 & $(90.8)$ & (7.7) \\
\hline Still in full-time education & 65 & 55 & $(84.6)$ & $(13.5)$ \\
\hline
\end{tabular}

\section{Ethnicity}

White

Non-white

\section{Country of birth\#}

UK

Outside UK

$4111 \quad 3227$

658

594

(77.0)

(90.0)

3668

969

2848

898

(75.9)

(92.7)

\section{9}

3194

(96.6)

(88.5)

Breast and formula milk

Formula milk only

Not sure

618

549

52

(6.4)

(67.4)

Gestation

$$
\begin{aligned}
& \text { 37-38 weeks } \\
& \text { 39-41 weeks }
\end{aligned}
$$

$\geq 42$ weeks

Type of birth

$$
\begin{aligned}
& \text { Normal vaginal } \\
& \text { Forceps/ventouse } \\
& \text { Planned caesarean } \\
& \text { Unplanned caesarean }
\end{aligned}
$$

\section{Duration of labour}

$$
\text { No labour }
$$$$
<8 \mathrm{~h}
$$

65

$819 \quad 616$

3484

515

3013

612

2406

551

584

522

1991
2837

$$
412
$$

500

418

(74.0)

(79.0)

(78.5)

(80.4)

(75.0)

(84.1)

493

393

1577
(74.3)

(77.8)
(14.9)

(4.3)

(4.3)
(29.6)

(15.7)

(21.0)

(15.6)

(17.7)

(20.9)

(19.2) 
Table 2 Breastfeeding initiation and cessation by socio-demographic, antenatal and birth characteristics (Continued)

\begin{tabular}{|c|c|c|c|c|c|}
\hline $8-17 \mathrm{~h}$ & 1215 & 992 & $(80.5)$ & $(13.7)$ & $(18.1)$ \\
\hline $18+h$ & 959 & 804 & (83.1) & (12.7) & $(18.0)$ \\
\hline \multicolumn{6}{|c|}{ Length of hospital stay } \\
\hline None (home birth) & 175 & 158 & (89.4) & (8.7) & $(10.1)$ \\
\hline$<24 \mathrm{~h}$ & 1612 & 1260 & $(76.9)$ & $(11.1)$ & $(15.3)$ \\
\hline 1-2 days & 1564 & 1265 & (79.8) & $(15.0)$ & $(18.2)$ \\
\hline 3-4 days & 1120 & 913 & (80.5) & $(14.3)$ & $(17.8)$ \\
\hline$\geq 5$ days & 139 & 107 & $(76.7)$ & (7.9) & $(19.3)$ \\
\hline \multicolumn{6}{|c|}{ Baby admitted to $\mathrm{NNU}^{\#}$} \\
\hline Yes & 296 & 216 & $(72.5)$ & (8.6) & $(18.9)$ \\
\hline No & 3851 & 3078 & (78.6) & (13.8) & (17.6) \\
\hline
\end{tabular}

${ }^{a}$ unweighted $n,{ }^{b} \%$ weighted for non-response, ${ }^{c}$ of those who initiated breastfeeding and reported timing of any breastfeeding cessation $(n=3840)$, ${ }^{d}$ of those who were still breastfeeding at 10 days and reported timing of any breastfeeding cessation $(n=3354)$.

\#all variables had $<3 \%$ missing data except for the following variables: country of birth ( $3 \%$ missing), length of hospital stay $(4 \%$ missing), baby NNU admission (15\% missing).

Table 3 Breastfeeding initiation and cessation by breastfeeding support factors

\begin{tabular}{|c|c|c|c|c|c|}
\hline & \multirow{2}{*}{$\begin{array}{l}\text { All women } \\
\qquad n^{\mathrm{a}}\end{array}$} & \multicolumn{2}{|c|}{$\begin{array}{l}\text { Initiated } \\
\text { breastfeeding }\end{array}$} & \multirow{2}{*}{$\begin{array}{c}\text { Stopped breastfeeding } \\
\text { by } 10 \text { days } \\
(\%)^{b, c}\end{array}$} & \multirow{2}{*}{$\begin{array}{c}\text { Stopped breastfeeding between } \\
10 \text { days and } 6 \text { weeks } \\
(\%)^{\text {b,d }}\end{array}$} \\
\hline & & $\mathrm{n}^{\mathrm{a}}$ & $(\%)^{\mathbf{b}}$ & & \\
\hline \multicolumn{6}{|c|}{$\begin{array}{l}\text { Feeding help/advice from parent support or } \\
\text { peer group }\end{array}$} \\
\hline Yes & 504 & 444 & $(86.9)$ & $(8.0)$ & $(13.0)$ \\
\hline No & 4310 & 3418 & $(78.2)$ & $(13.6)$ & $(17.2)$ \\
\hline \multicolumn{6}{|c|}{ Feeding help/advice from voluntary organisation } \\
\hline Yes & 264 & 257 & $(97.3)$ & $(5.1)$ & $(12.7)$ \\
\hline No & 4550 & 3605 & $(78.1)$ & $(12.7)$ & $(17.1)$ \\
\hline \multicolumn{6}{|c|}{ Feeding help/advice from breastfeeding clinic } \\
\hline Yes & 584 & 572 & $(97.8)$ & $(5.0)$ & $(15.4)$ \\
\hline No & 4230 & 3290 & $(76.6)$ & $(13.6)$ & $(17.0)$ \\
\hline \multicolumn{6}{|c|}{ Needed help and advice re. feeding } \\
\hline Yes & 3315 & 617 & $(60.3)$ & $(14.2)$ & $(18.1)$ \\
\hline No & 882 & 547 & $(83.4)$ & $(5.5)$ & $(9.4)$ \\
\hline \multicolumn{6}{|l|}{ Attended a baby café } \\
\hline Yes & 283 & 239 & $(82.5)$ & $(9.4)$ & $(10.4)$ \\
\hline No & 874 & 689 & $(77.4)$ & $(12.0)$ & $(16.4)$ \\
\hline Service not available & 3595 & 2882 & $(79.2)$ & $(13.6)$ & $(17.4)$ \\
\hline \multicolumn{6}{|c|}{$\begin{array}{l}\text { Midwives gave active support and encouragement } \\
\text { re. feeding }\end{array}$} \\
\hline Yes, always & 1861 & 1588 & $(84.4)$ & $(11.3)$ & $(14.3)$ \\
\hline Yes, generally & 1927 & 1603 & $(82.1)$ & $(13.3)$ & $(18.4)$ \\
\hline No & 727 & 489 & $(65.6)$ & $(19.6)$ & $(21.5)$ \\
\hline Don't know & 85 & 58 & $(67.4)$ & $(7.0)$ & $(15.8)$ \\
\hline Didn't want this & 150 & 75 & (48.7) & (6.6) & (6.0) \\
\hline
\end{tabular}

${ }^{a}$ unweighted $\mathrm{n},{ }^{\mathrm{b}} \%$ weighted for non-response, ${ }^{c}$ of those who initiated breastfeeding and reported timing of any breastfeeding cessation $(n=3840),{ }^{d}$ of those who were still breastfeeding at 10 days and reported timing of any breastfeeding cessation $(n=3354)$. 
Table 4 Socio-demographic, antenatal and birth factors associated with breastfeeding cessation in multivariable analysis

\begin{tabular}{|c|c|c|c|c|c|c|c|c|}
\hline & \multicolumn{4}{|c|}{ Breastfeeding cessation by 10 days } & \multicolumn{4}{|c|}{$\begin{array}{l}\text { Breastfeeding cessation between } \\
10 \text { days and } 6 \text { weeks }\end{array}$} \\
\hline & \multicolumn{2}{|c|}{$\begin{array}{l}\text { CRUDE OR (complete } \\
\text { case) }(n=3451)\end{array}$} & \multicolumn{2}{|c|}{$\begin{array}{c}\text { FINAL ADJUSTED } \\
\text { (complete case) }(n=3451)\end{array}$} & \multicolumn{2}{|c|}{$\begin{array}{l}\text { CRUDE OR (complete } \\
\text { case) }(n=2980)\end{array}$} & \multicolumn{2}{|c|}{$\begin{array}{c}\text { FINAL ADJUSTED } \\
\text { (complete case) }(n=2980)\end{array}$} \\
\hline & OR & $(95 \% \mathrm{Cl})$ & OR & $(95 \% \mathrm{Cl})$ & OR & $(95 \% \mathrm{Cl})$ & OR & $(95 \% \mathrm{Cl})$ \\
\hline \multicolumn{9}{|l|}{ Index of Multiple Deprivation } \\
\hline Quintile 1 (least deprived) & 1.00 & - & 1.00 & - & 1.00 & - & 1.00 & - \\
\hline Quintile & 1.20 & $(0.86,1.66)$ & 1.07 & $(0.75,1.54)$ & 1.15 & $(0.85,1.57)$ & 1.13 & $(0.82,1.57)$ \\
\hline Quintile 3 & $1.46^{*}$ & $(1.07,2.01)$ & 1.38 & $(0.99,1.93)$ & $1.49^{* *}$ & $(1.11,2.01)$ & $1.57^{* *}$ & $(1.15,2.16)$ \\
\hline Quintile 4 & $1.53^{*}$ & $(1.10,2.12)$ & $1.50^{*}$ & $(1.03,2.18)$ & $1.44^{*}$ & $(1.06,1.97)$ & $1.45^{*}$ & $(1.03,2.03)$ \\
\hline Quintile 5 (most deprived) & $1.75^{* * *}$ & $(1.26,2.43)$ & $1.84^{* *}$ & $(1.27,2.68)$ & $1.55^{* *}$ & $(1.13,2.14)$ & $1.79^{* *}$ & $(1.25,2.57)$ \\
\hline \multicolumn{9}{|l|}{ Age } \\
\hline $16-19$ & $3.84^{* * *}$ & $(2.15,6.85)$ & 1.9 & $(0.97,3.74)$ & $3.32^{* * *}$ & $(1.69,6.53)$ & 1.84 & $(0.93,3.63)$ \\
\hline $20-24$ & $2.39^{* * *}$ & $(1.75,3.27)$ & $1.51^{*}$ & $(1.04,2.19)$ & $1.73^{* *}$ & $(1.24,2.41)$ & 1.24 & $(0.85,1.79)$ \\
\hline $25-29$ & 1.22 & $(0.93,1.59)$ & 1.12 & $(0.83,1.50)$ & 1.08 & $(0.84,1.39)$ & 1.02 & $(0.78,1.33)$ \\
\hline $30-34$ & 1.00 & - & 1.00 & - & 1.00 & - & 1.00 & - \\
\hline $35-39$ & $0.66^{*}$ & $(0.48,0.91)$ & $0.66^{*}$ & $(0.48,0.92)$ & $0.58^{* * *}$ & $(0.43,0.77)$ & $0.55^{* * *}$ & $(0.40,0.75)$ \\
\hline$\geq 40$ & 0.70 & $(0.41,1.19)$ & 0.64 & $(0.37,1.13)$ & 0.61 & $(0.36,1.01)$ & $0.52^{*}$ & $(0.30,0.89)$ \\
\hline \multicolumn{9}{|c|}{ Age completed full-time education } \\
\hline$<17$ years & $3.35^{* * *}$ & $(2.57,4.36)$ & $2.15^{* * *}$ & $(1.58,2.92)$ & $3.44^{* * *}$ & $(2.66,4.46)$ & $3.03^{* * *}$ & $(2.29,4.02)$ \\
\hline $17-18$ years & $2.92^{* * *}$ & $(2.29,3.73)$ & $1.99^{* * *}$ & $(1.52,2.61)$ & $2.59^{* * *}$ & $(2.05,3.27)$ & $2.24^{* * *}$ & $(1.74,2.87)$ \\
\hline $19+$ years & 1.00 & - & 1.00 & - & 1.00 & - & 1.00 & - \\
\hline Still in full-time education & 1.89 & $(0.79,4.55)$ & 2.11 & $(0.86,5.22)$ & 1.21 & $(0.46,3.22)$ & 1.31 & $(0.45,3.81)$ \\
\hline \multicolumn{9}{|l|}{ Ethnicity } \\
\hline White & $3.47^{* * *}$ & $(2.22,5.43)$ & $3.02^{* * *}$ & $(1.74,5.22)$ & $2.48^{* * *}$ & $(1.73,3.54)$ & $2.49^{* * *}$ & $(1.63,3.80)$ \\
\hline Non-white & 1.00 & - & 1.00 & - & 1.00 & - & 1.00 & - \\
\hline \multicolumn{9}{|l|}{ Country of birth } \\
\hline UK & $4.29^{* * *}$ & $(2.89,6.36)$ & $2.91^{* * *}$ & $(1.85,4.56)$ & $2.58^{* * *}$ & $(1.92,3.46)$ & $1.71^{* *}$ & $(1.63,3.06)$ \\
\hline Outside UK & 1.00 & - & 1.00 & - & 1.00 & - & 1.00 & - \\
\hline \multicolumn{9}{|l|}{ Antenatal feeding intention } \\
\hline Breast milk only & 1.00 & - & 1.00 & - & 1.00 & - & 1.00 & - \\
\hline Breast and formula milk & $2.06^{* * *}$ & $(1.58,2.67)$ & $2.47^{* * *}$ & $(1.84,3.31)$ & $2.03^{* * *}$ & $(1.56,2.63)$ & $2.63^{* * *}$ & $(1.97,3.53)$ \\
\hline Formula milk only & $8.70^{* * *}$ & $(4.82,15.71)$ & $6.90^{* * *}$ & $(2.94,16.20)$ & $6.18^{* * *}$ & $(2.71,14.10)$ & $4.73^{* *}$ & $(1.74,12.87)$ \\
\hline Not sure & $4.10^{* * *}$ & $(2.31,7.28)$ & $4.01^{* * *}$ & $(2.17,7.42)$ & 1.33 & $(0.57,3.09)$ & 1.09 & $(0.45,2.63)$ \\
\hline
\end{tabular}

\section{Duration of labour}

$\begin{array}{lcccc}\begin{array}{l}\text { No labour } \\ <8 \mathrm{~h}\end{array} & 0.66^{*} & (0.44,0.98) & 0.55^{* *} & (0.36,0.84) \\ 8-17 \mathrm{~h} & 1.00 & - & 1.00 & - \\ \begin{array}{l}\text { 18+ } \mathrm{h} \\ \text { ength of hospital stay }\end{array} & 1.03 & (0.79,1.28) & 0.97 & (0.73,1.29) \\ \text { None (home birth) } & 0.98 & (0.72,1.21) & 0.89 & (0.66,1.22) \\ <24 \mathrm{~h} & & & & \\ \text { 1-2 days } & 0.78 & (0.43,1.41) & 0.88 & (0.46,1.71) \\ \text { 3-4 days } & 1.00 & - & 1.00 & - \\ \geq 5 \text { days } & 1.39^{*} & (1.08,1.78) & 1.42^{*} & (1.08,1.88) \\ & 1.32 & (1.00,1.74) & 1.78^{* * *} & (1.29,2.46) \\ & 0.66 & (0.31,1.41) & 1.39 & (0.59,3.24)\end{array}$


Table 4 Socio-demographic, antenatal and birth factors associated with breastfeeding cessation in multivariable analysis (Continued)

\begin{tabular}{|c|c|c|c|c|c|c|c|c|}
\hline \multicolumn{9}{|l|}{ Gestation } \\
\hline 37-38 weeks & - & - & - & - & 1.21 & $(0.93,1.59)$ & 1.25 & $(0.93,1.68)$ \\
\hline 39-41 weeks & - & - & - & - & 1.00 & - & 1.00 & - \\
\hline$\geq 42$ weeks & - & - & - & - & $1.58^{* *}$ & $(1.17,2.12)$ & $1.40^{*}$ & $(1.01,1.95)$ \\
\hline \multicolumn{9}{|l|}{ Type of birth } \\
\hline Normal vaginal & - & - & - & - & 1.00 & - & 1.00 & - \\
\hline Forceps/ventouse & - & - & - & - & 1.19 & $(0.89,1.60)$ & 1.14 & $(0.84,1.54)$ \\
\hline Planned caesarean & - & - & - & - & $1.42^{*}$ & $(1.05,1.91)$ & $1.50^{*}$ & $(1.09,2.07)$ \\
\hline Unplanned caesarean & - & - & - & - & 1.24 & $(0.91,1.67)$ & 1.30 & $(0.93,1.81)$ \\
\hline \multicolumn{9}{|l|}{ Baby admitted to NNU } \\
\hline Yes & $0.55^{*}$ & $(0.32,0.93)$ & $0.42^{* *}$ & $(0.23,0.78)$ & - & - & - & - \\
\hline No & 1.00 & - & 1.00 & - & - & - & - & - \\
\hline
\end{tabular}

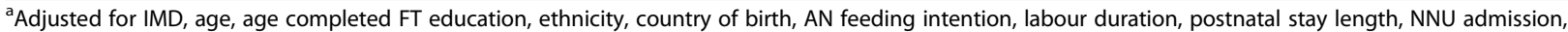
source of breastfeeding support, breastfeeding support not needed, MWs gave active support and encouragement.

${ }^{b}$ Adjusted for IMD, age, age completed FT education, ethnicity, country of birth, AN feeding intention, gestation, type of birth, baby café attendance, breastfeeding support not needed, MWs gave active support and encouragement.

*p $<0.05{ }^{* *} p<0.005 * * *<0.001$.

women who did not initiate breastfeeding, making it difficult to isolate a similar association. However, a small number of studies have focused only on those women who initiated breastfeeding, and these have reported similar socio-demographic trends to those observed here $[6,8,9]$. The socio-demographic factors considered here are unmodifiable; nevertheless our understanding of these associations is key to the design and targeting of appropriate interventions.

The role of antenatal feeding intention was very strongly associated with breastfeeding in our analysis. In an analysis of data from a maternity survey conducted in England in 2006, a strong effect of antenatal feeding intention on breastfeeding outcomes was observed, [4] with similar associations reported in other studies $[2,3,5]$. Less than one in five women in this analysis reported intending to formula feed (17\%) and few reported being undecided (2\%), though these figures may be underestimates given that women may be more likely to retrospectively declare an intention to breastfeed. Feeding intention is known to be associated with sociodemographic characteristics, and is also likely to be strongly correlated with peer infant feeding behaviour and whether the mother herself was breastfed. These two latter factors were identified as predictors of breastfeeding continuation in a recent analysis of 2010 IFS data [1].

We explored the role of breastfeeding support on cessation by 10 days and by six weeks. In our survey women were asked whether they had received feeding help from a number of different sources. Women who received help from a parent or peer support group, voluntary organisation, or a breastfeeding clinic were all less likely to have stopped breastfeeding at 10 days, though there was no significant association between these factors and cessation at six weeks. Our findings confirm that even after adjusting for other factors (including the need for support), the provision of breastfeeding support, particularly from non-health professionals, may have an important role in reducing the number of women who stop breastfeeding in the first few weeks. This finding was supported by substantial PAFs, for example estimating that $59 \%$ of breastfeeding cessations are attributable to women who need help with feeding not receiving help and advice from a voluntary organisation. As discussed previously, although descriptive studies have linked breastfeeding support to increases in breastfeeding continuation particularly in the early weeks, $[1,21]$ results from UK trials have generally reported no effect on breastfeeding outcomes, even where peer support has been considered as a stand-alone intervention [16]. In an observational study such as this, it is likely that motivation to seek support partly explains the association between breastfeeding support and breastfeeding cessation. The type of support considered here (from non-health professionals) relies on women proactively seeking support: women with high intention or commitment to breastfeed may be more likely to seek help when problems arise.

A number of birth factors were independently associated with breastfeeding cessation at 10 days and/or 6 weeks. Of the observed associations, one particularly interesting trend was seen for caesareans, in particular planned caesareans which were associated with a higher odds of cessation at 6 weeks. Information was not collected 
Table 5 Breastfeeding support factors associated with breastfeeding cessation in multivariable analysis

\begin{tabular}{|c|c|c|c|c|c|c|c|c|c|c|}
\hline & \multicolumn{6}{|c|}{ Breastfeeding cessation by 10 days } & \multicolumn{4}{|c|}{$\begin{array}{l}\text { Breastfeeding cessation between } \\
10 \text { days and } 6 \text { weeks }\end{array}$} \\
\hline & \multicolumn{2}{|c|}{$\begin{array}{c}\text { CRUDE OR } \\
\text { (complete case) } \\
(n=3451) \\
\end{array}$} & \multicolumn{2}{|c|}{$\begin{array}{c}\text { FINAL ADJUSTED } \\
\text { (complete case) } \\
(n=3451) \\
\end{array}$} & \multirow[b]{2}{*}{ PAF } & \multirow[b]{2}{*}{$95 \% \mathrm{Cl}$} & \multicolumn{2}{|c|}{$\begin{array}{c}\text { CRUDE OR } \\
\text { (complete case) } \\
(n=2980) \\
\end{array}$} & \multicolumn{2}{|c|}{$\begin{array}{c}\text { FINAL ADJUSTED } \\
\text { (complete case) } \\
(n=2980)\end{array}$} \\
\hline & OR & $(95 \% \mathrm{Cl})$ & OR & $(95 \% \mathrm{Cl})$ & & & OR & $(95 \% \mathrm{Cl})$ & OR & $(95 \% \mathrm{Cl})$ \\
\hline \multicolumn{11}{|l|}{ Attended a baby café } \\
\hline Yes & - & - & - & - & - & - & 1.00 & - & 1.00 & - \\
\hline No & - & - & - & - & - & - & $1.68^{*}$ & $(1.02,2.77)$ & 1.47 & $(0.87,2.50)$ \\
\hline Service not available & - & - & - & - & - & - & $1.80^{*}$ & $(1.14,2.86)$ & $1.73^{*}$ & $(1.06,2.81)$ \\
\hline \multicolumn{11}{|c|}{$\begin{array}{l}\text { Feeding help/advice from parent support } \\
\text { or peer group }\end{array}$} \\
\hline Yes & 1.00 & - & 1.00 & - & & & - & - & & \\
\hline No & $1.67^{* *}$ & $(1.14,2.46)$ & $1.58^{*}$ & $(1.03,2.41)$ & $34.0 \%$ & $(2.7-55.2 \%)$ & - & - & - & - \\
\hline \multicolumn{11}{|c|}{$\begin{array}{l}\text { Feeding help/advice from voluntary } \\
\text { organisation }\end{array}$} \\
\hline Yes & 1.00 & - & 1.00 & - & & & - & - & - & - \\
\hline No & $2.72^{* * *}$ & $(1.84,4.02)$ & $2.65^{* * *}$ & $(1.76,3.98)$ & $58.1 \%$ & $(39.1-71.2 \%)$ & - & - & - & - \\
\hline \multicolumn{11}{|c|}{ Needed help and advice re. feeding } \\
\hline Yes & $2.79^{* * *}$ & $(1.85,4.20)$ & $2.84^{* * *}$ & $(1.83,4.40)$ & - & - & $2.36^{* * *}$ & $(1.66,3.36)$ & $2.32^{* * *}$ & $(1.58,3.40)$ \\
\hline No & 1.00 & - & 1.00 & - & - & - & 1.00 & - & 1.00 & - \\
\hline \multicolumn{11}{|c|}{$\begin{array}{l}\text { Midwives gave active support and } \\
\text { encouragement re. feeding }\end{array}$} \\
\hline Yes, always & 1.00 & - & 1.00 & - & - & - & 1.00 & - & 1.00 & - \\
\hline Yes, generally & 1.22 & $(0.97,1.54)$ & $1.34^{*}$ & $(1.04,1.72)$ & - & - & $1.34^{* *}$ & $(1.08,1.67)$ & $1.58^{* * *}$ & $(1.25,1.99)$ \\
\hline No & $1.97^{* * *}$ & $(1.47,2.64)$ & $2.22^{* * *}$ & $(1.59,3.11)$ & - & - & $1.73^{* * *}$ & $(1.28,2.34)$ & $2.01^{* * *}$ & $(1.45,2.77)$ \\
\hline Don't know & 0.54 & $(0.16,1.83)$ & 0.57 & $(0.15,2.09)$ & - & - & 0.80 & $(0.33,1.92)$ & 0.88 & $(0.39,2.02)$ \\
\hline Didn't want this & 0.46 & $(0.14,1.50)$ & 1.16 & $(0.37,3.71)$ & - & - & $0.30^{*}$ & $(0.09,0.98)$ & 0.71 & $(0.23,2.22)$ \\
\hline
\end{tabular}

${ }^{a}$ Adjusted for IMD, age, age completed FT education, ethnicity, country of birth, AN feeding intention, labour duration, postnatal stay length, NNU admission, source of breastfeeding support, breastfeeding support not needed, MWs gave active support and encouragement.

${ }^{\mathrm{b}}$ Adjusted for IMD, age, age completed FT education, ethnicity, country of birth, AN feeding intention, gestation, type of birth, baby café attendance, breastfeeding support not needed, MWs gave active support and encouragement. * $p<0.05 * * p<0.005 * * * p<0.001$.

on the reasons for caesarean section (maternal conditions, fetal conditions, or other) so it is difficult to suggest possible explanations for this trend. NNU admission was associated with a lower odds of cessation at 10 days, probably due to the fact many of these mothers may have been given support with feeding whilst their babies received care.

\section{Strengths and limitations}

Overall our study was large, though our response rate was relatively low (55\%) and non-responders differed from responders in terms of a number of factors, most notably, IMD quintile [17]. We addressed this bias by weighting the analysis according to IMD quintile. This approach, together with the fact that our figures on breastfeeding prevalence are similar to those reported in the IFS from the same time period, [1] suggests our survey is fairly representative.
In our analysis we had access to data on a wide range of factors potentially associated with breastfeeding. Due to the survey design we were unable to look at exclusivity of breastfeeding beyond the first few days. In addition, information was not collected on previous feeding experiences; previous breastfeeding experience has found to be a predictor of breastfeeding duration in other studies [22] and it is likely to be also associated with the need for breastfeeding support.

The focus of the study was maternity care and the survey was conducted when infants were approximately three months old. Although women were explicitly asked about care from midwives and also from health professionals more generally, we did not ask about support provided by other specific health professionals such as health visitors. Due to the timing of the survey we were unable to investigate longer-term breastfeeding, 
which is potentially subject to influence from different factors. However, the fact that the survey was carried out early in the postnatal period should have minimised bias in the recall of breastfeeding behaviour and other factors relating to the antenatal and birth period.

Women in our survey were asked to report on their use of breastfeeding support services but we did not seek information on the timing of this. Therefore, misclassification may have occurred in the analysis of 10 day cessation as women who were classified as receiving help from a particular source may have in fact sought help beyond 10 days.

In calculating PAFs, we have assumed causality. However, the relationship between breastfeeding support and cessation is clearly complex, and as discussed earlier, must be considered in the context of other factors such as motivation to seek help and support. Nevertheless, calculating PAFs enables us to consider the potential maximum effect of improving breastfeeding support and can be considered a useful addition to the research evidence.

\section{Implications}

In 2011 there were 688,120 births in England [23]. Assuming the initiation rate (79\%), and the 10 day (68\%) and six week (57\%) continuation rates observed in our study, we estimate that approximately 151,000 mothers who started breastfeeding in 2011 stopped by the time their baby was six weeks old. This represents a considerable number of breastfeeding cessations that could potentially be prevented. Of those mothers questioned in the 2010 IFS who had stopped breastfeeding, nearly two-thirds said they would have liked to have breastfed for longer [1].

Multiple factors influence a mother's likelihood of continuing breastfeeding. These factors clearly need to be addressed by interventions which address the socio-demographic context in which a mother breastfeeds. Despite the lack of effect observed in previous evaluations of breastfeeding interventions in the UK, breastfeeding support remains a central component of addressing low breastfeeding rates, and efforts need to be focused on the most appropriate type of support in terms of timing, intensity and delivery. Our results suggest that breastfeeding support delivered by non-health professionals (peer support, voluntary organisation) and specialist support such as breastfeeding clinics may have an important role in preventing breastfeeding cessations in the first few weeks among women giving birth to term singletons.

\section{Conclusions}

A substantial proportion of women who initiate breastfeeding are no longer breastfeeding at six weeks. Efforts to improve breastfeeding rates in the UK are unlikely to be successful without a strong evidence base regarding the specific factors which are associated with breastfeeding continuation and an attempt to address these factors through tailored interventions. Despite the negative findings from UK trials of breastfeeding support, our results suggest that breastfeeding support may help to prevent breastfeeding cessation in the first few weeks. There is a clear need for further research in order to identify the most appropriate interventions for increasing breastfeeding duration.

\section{Additional file}

Additional file 1: Table S1. Breastfeeding initiation and cessation by additional explanatory factors not associated with breastfeeding cessation in multivariable analysis.

\section{Competing interests}

The authors declare that they have no competing interests.

\section{Authors' contributions}

All authors contributed to the analysis plan, and LO conducted the analysis with guidance from MQ. $L O$ and MQ wrote the initial draft of the manuscript. All authors contributed to critical review and approved the final version of the manuscript.

\section{Funding}

This paper reports on an independent study which is funded by the Policy Research Programme in the Department of Health. The views expressed are not necessarily those of the Department. The funder had no role in: the design and conduct of the study; the analysis and interpretation of the data; and the drafting of the paper and decision to submit the paper for publication.

Received: 4 September 2013 Accepted: 14 February 2014

Published: 26 February 2014

\section{References}

1. Health and Social Care Information Centre, IFF Research: Infant Feeding Survey 2010. Leeds: Health and Social Care Information Centre; 2012.

2. Forster D, McLachlan H, Lumley J: Factors associated with breastfeeding at six months postpartum in a group of Australian women. Int Breastfeed J 2006, 1(1):1-12.

3. Donath SM, Amir LH, The Alspac Study Team: Relationship between prenatal infant feeding intention and initiation and duration of breastfeeding: a cohort study. Acta Paediatr 2003, 92(3):352-356.

4. Henderson J, Redshaw M: Midwifery factors associated with successful breastfeeding. Child Care Health Dev 2011, 37(5):744-753.

5. Scott JA, Binns CW, Oddy WH, Graham Kl: Predictors of breastfeeding duration: evidence from a cohort study. Pediatrics 2006, 117(4):e646-e655.

6. Agboado G, Michel E, Jackson E, Verma A: Factors associated with breastfeeding cessation in nursing mothers in a peer support programme in Eastern Lancashire. BMC Pediatr 2010, 10(1):3.

7. Kelly YJ, Watt RG, Nazroo JY: Racial/ethnic differences in breastfeeding initiation and continuation in the United Kingdom and comparison with findings in the United States. Pediatrics 2006, 118(5):e1428-e1435.

8. Baxter J, Cooklin AR, Smith J: Which mothers wean their babies prematurely from full breastfeeding? An Australian cohort study. Acta Paediatr 2009, 98(8):1274-1277.

9. Wright CM, Parkinson K, Scott J: Breast-feeding in a UK urban context: who breast-feeds, for how long and does it matter? Public Health Nutr 2006, 9(06):686-691.

10. Tarrant RC, Younger KM, Sheridan-Pereira M, Kearney JM: Factors associated with duration of breastfeeding in Ireland: potential areas for improvement. J Hum Lactation 2011, 27(3):262-271. 
11. Brown $A E$, Raynor $P$, Benton $D$, Lee MD: Indices of multiple deprivation predict breastfeeding duration in England and Wales. Eur $\mathrm{J}$ Public Health 2010, 20(2):231-235.

12. Amir $L H$, Donath $S M$ : Socioeconomic status and rates of breastfeeding in Australia: evidence from three recent national health surveys. Med J Aust 2008, 189(5):254-256.

13. Prior E, Santhakumaran S, Gale C, Philipps LH, Modi N, Hyde MJ: Breastfeeding after cesarean delivery: a systematic review and metaanalysis of world literature. Am J Clin Nutr 2012, 95(5):1113-1135.

14. Renfrew MJ, McCormick FM, Wade A, Quinn B, Dowswell T: Support for healthy breastfeeding mothers with healthy term babies. Cochrane Database Syst Rev 2012. Issue 5. Art. No.: CD001141. DOl: 10.1002/14651858. CD001141.pub4.

15. Hoddinott P, Seyara R, Marais D: Global evidence synthesis and UK idiosyncrasy: why have recent UK trials had no significant effects on breastfeeding rates? Matern Child Nutr 2011, 7(3):221-227.

16. Jolly K, Ingram L, Khan KS, Deeks JJ, Freemantle N, MacArthur C: Systematic review of peer support for breastfeeding continuation: metaregression analysis of the effect of setting, intensity, and timing. BMJ 2012, 344:d8287.

17. Redshaw M, Heikkila K: Delivered with care: A national survey of women's experience of maternity care 2010. Oxford: National Perinatal Epidemiology Unit, University of Oxford; 2010.

18. McLennan D, Barnes H, Noble M, Davies J, Garratt E, Dibben C: The English Indices of Deprivation 2010. London: Department for Communities and Local Government; 2011

19. Greenland S: Re:"Confidence limits made easy: interval estimation using a substitution method". Am J Epidemiol 1999, 149(9):884.

20. Oakley LL, Renfrew MJ, Kurinczuk JJ, Quigley MA: Factors associated with breastfeeding in England: an analysis by primary care trust. BMJ Open 2013, 3:e002765. doi:10.1136/bmjopen-2013-002765.

21. Bartington $S$, Griffiths $\sqcup$, Tate AR, Dezateux C, Group tMCSCH: Are breastfeeding rates higher among mothers delivering in Baby Friendly accredited maternity units in the UK? Int J Epidemiol 2006, 35(5):1178-1186.

22. Kronborg $\mathrm{H}$, Væth $\mathrm{M}$ : The influence of psychosocial factors on the duration of breastfeeding. Scand J Public Healt 2004, 32(3):210-216.

23. Birth Summary Tables - England and Wales, 2011 (Final): Birth Summary Tables - England and Wales, 2011 (Final). http://www.ons.gov.uk/ons/rel/ vsob1/birth-summary-tables-england-and-wales/2011-final-/index.html.

doi:10.1186/1471-2393-14-88

Cite this article as: Oakley et al:: The role of support and other factors in early breastfeeding cessation: an analysis of data from a maternity survey in England. BMC Pregnancy and Childbirth 2014 14:88.

\section{Submit your next manuscript to BioMed Central and take full advantage of:}

- Convenient online submission

- Thorough peer review

- No space constraints or color figure charges

- Immediate publication on acceptance

- Inclusion in PubMed, CAS, Scopus and Google Scholar

- Research which is freely available for redistribution

Submit your manuscript at www.biomedcentral.com/submit 